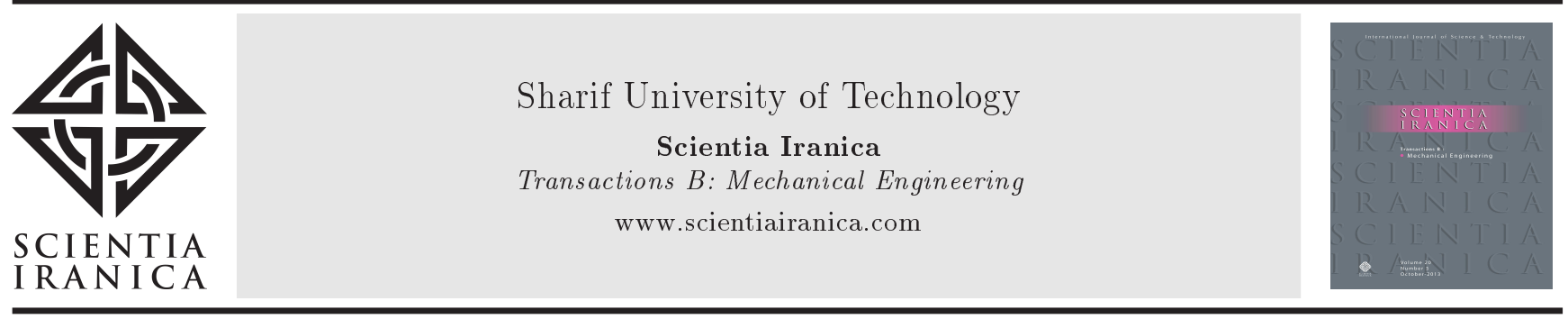

Research Note

\title{
Non-axisymmetric stagnation-point flow and heat transfer of a viscous fluid on a stationary cylinder
}

\author{
R. Alizadeh ${ }^{\mathrm{a}}$, A.B. Rahimi ${ }^{\mathrm{b}, *}$ and M. Najafi ${ }^{\mathrm{a}}$ \\ a. Department of Mechanical \& Aerospace Engineering, Tehran Science and Research Branch, Islamic Azad University, Tehran, \\ Iran. \\ b. Faculty of Engineering, Ferdowsi University of Mashhad, Mashhad, P.O. Box 917r5-1111, Iran.
}

Received 3 September 2014; received in revised form 9 June 2015; accepted 12 October 2015

\section{KEYWORDS}

Non-axisymmetric stagnation-point flow;

Heat transfer;

Stationary cylinder;

Viscous fluid;

Numerical solution;

Non-uniform

transpiration.

\begin{abstract}
The steady-state viscous flow and heat transfer in the vicinity of a nonaxisymmetric stagnation point of an infinite stationary cylinder with non-uniform normal transpiration, $U_{0}(\varphi)$, and constant wall temperature are investigated. The impinging free stream is steady and with a constant strain rate, $\bar{k}$. A reduction in Navier-Stokes and energy equations is obtained by use of appropriate similarity transformations. The semisimilar solution of the Navier-Stokes equations and energy equation has been obtained numerically using an implicit finite-difference scheme. All the solutions above are presented for Reynolds number, Re $=\bar{k} a^{2} / 2 v$, ranging from 0.01 to 100 for different values of Prandtl number and for selected values of transpiration rate function, $S(\varphi)=U_{0}(\varphi) / \bar{k} a$, where $a$ is cylinder radius and $v$ is kinematic viscosity of the fluid. Dimensionless shear stresses corresponding to all the cases increase with increase in Reynolds number and transpiration rate function. The local coefficient of heat transfer (Nusselt number) increases with the increasing transpiration rate function and Prandtl number.
\end{abstract}

(C) 2016 Sharif University of Technology. All rights reserved.

\section{Introduction}

The effects of non-uniform normal transpiration on a stationary cylinder with different types of transpiration rate function, which are of interest in certain special manufacturing processes, textile technology, printing industry, water-show technology, cooling and centrifugal processes in industry, and calcinations of cement, have not yet been considered. Our motivation is originally to produce frictionless situations in fluids and insulated surfaces in heat transfer by means of certain types of movements and temperature difference. Since

*. Corresponding author. Tel.: +985138805018

E-mail addresses: R_alizadeh86@yahoo.com (R. Alizadeh); rahimiab@yahoo.com (A.B.Rahimi); M.njafi36@gmail.com (M. Najafi) the decay of viscosity in a fluid and diffusion of heat in a medium are naturally exponential, selection of the corresponding function in this study serves appropriate examples. Besides, our study generalizes the problem of stagnation-point flow and heat transfer of a fluid on a stationary cylinder.

There are many solutions of the Navier-Stockes and energy equations regarding the problem of stagnation-point flow and heat transfer in the vicinity of a flat plate or a cylinder. These studies started by Hiemenz [1] who obtained an exact solution of the Navier-Stokes equations governing the two-dimensional stagnation-point flow on a flat plate, and were continued by Homann [2] with an analogous axisymmetric study and by Howarth [3] and Davey [4], whose results for stagnation-point flow against a flat plate for asymmetric cases were presented. Wang [5,6] was 
the first to find an exact solution for the problem of axisymmetric stagnation-point flow on an infinite stationary circular cylinder; this was continued by Gorla's works [7-11], which are a series of steady and unsteady flows and heat transfer over a circular cylinder in the vicinity of the stagnation point for the cases of constant axial movement and the special case of axial harmonic motion of a non-rotating cylinder. Cunning et al. [12] have considered the stagnationpoint flow problem on a rotating circular cylinder with constant angular velocity; Grosch and Salwen [13] as well as Takhar et al. [14] studied special cases of unsteady viscous flow on an infinite circular cylinder. The most recent works of the same types are the ones by Saleh and Rahimi [15] and Rahimi and Saleh [16,17], which are exact solution studies of a stagnation-point flow and heat transfer on a circular cylinder with timedependent axial and rotational movements, as well as studies by Abbasi and Rahimi [18-21], which are exact solutions of stagnation-point flow and heat transfer but on a flat plate. Some existing compressible flow studies, but in the stagnation region of bodies and by using boundary layer equations, include the study by Subhashini and Nath [22] as well as Kumari and Nath [23,24], which are in the stagnation region of a body, and the work by Katz [25] as well as those of Afzal and Ahmad [26], Libby [27], and Gersten et al. [28], which are all general studies in the stagnation region of a body. Recently, Mohammadiun and Rahimi [29] and Mohammadiun et al. [30] considered the stagnation-point flow and heat transfer of a viscous, compressible fluid problem on a stationary circular cylinder.

All the studies mentioned above consider the axisymmetric flow and heat transfer and none has considered the effect of flow being non-axisymmetric, except [31] which is for the moving cylinder. In the present analysis, the problem of non-axisymmetric stagnation-point flow and heat transfer of a viscous fluid on a stationary cylinder with non-uniform transpiration and constant wall temperature are considered for the first time. A reduction in Navier-Stokes and energy equations is obtained by use of appropriate similarity transformations. The semi-similar solution of these equations is obtained numerically using an implicit finite-difference scheme. All the solutions above are presented for Reynolds number, $\operatorname{Re}=\bar{k} a^{2} / 2 v$, ranging from 0.01 to 100 for different values of Prandtl number and for selected values of transpiration rate function, $S(\varphi)=U_{0}(\varphi) / \bar{k} a$, where $a$ is cylinder radius and $v$ is kinematic viscosity of the fluid. Dimensionless shearstresses corresponding to all the cases increase with increase in Reynolds number and transpiration rate function. The local coefficient of heat transfer (Nusselt number) increases with increasing transpiration rate function and Prandtl number.
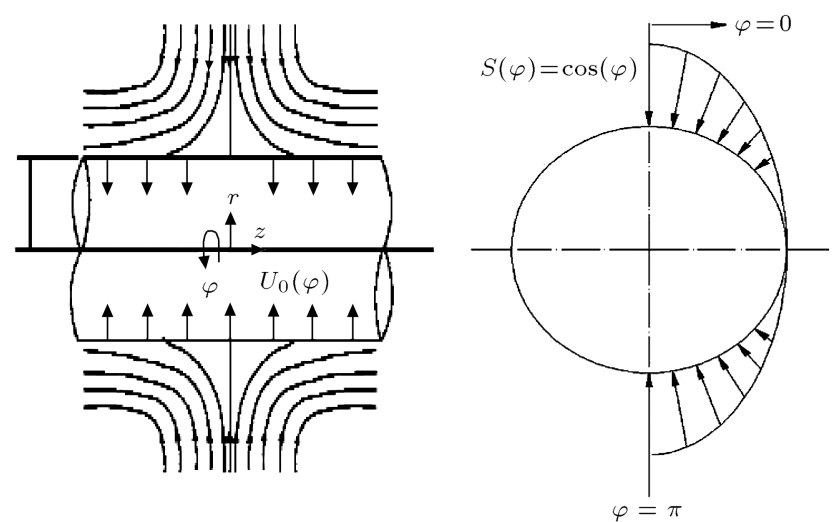

Figure 1. Schematic diagram of a stationary cylinder under radial stagnation flow.

\section{Problem formulation}

Flow is considered in cylindrical coordinates $(r, \varphi, z)$ with corresponding velocity components $(u, v, w)$; see Figure 1. An external axisymmetric radial stagnationpoint flow of strain rate $\bar{k}$ impinges on the cylinder of radius $a$, centered at $r=0$. Because of existence of a selected transpiration function shown below, a laminar steady incompressible flow is formed and heat transfer of a viscous fluid happens in the neighborhood of a nonaxisymmetric stagnation point of a stationary infinite circular cylinder with constant wall temperature.

The steady Navier-Stokes and energy equations in cylindrical polar coordinates governing the nonaxisymmetric incompressible flow and heat transfer are as follows [32]:

Mass:

$$
\frac{\partial u}{\partial r}+\frac{u}{r}+\frac{\partial w}{\partial z}=0
$$

$r$-momentum:

$$
\begin{aligned}
u \frac{\partial u}{\partial r} & +w \frac{\partial u}{\partial z}=-\frac{1}{\rho} \frac{\partial p}{\partial r} \\
& +v\left[\frac{\partial^{2} u}{\partial r^{2}}+\frac{1}{r} \frac{\partial u}{\partial r}-\frac{u}{r^{2}}+\frac{1}{r^{2}} \frac{\partial^{2} u}{\partial \varphi^{2}}+\frac{\partial^{2} u}{\partial z^{2}}\right]
\end{aligned}
$$

$z$-momentum:

$$
\begin{aligned}
u \frac{\partial w}{\partial r} & +w \frac{\partial w}{\partial z}=-\frac{1}{\rho} \frac{\partial p}{\partial z} \\
& +v\left[\frac{\partial^{2} w}{\partial r^{2}}+\frac{1}{r} \frac{\partial w}{\partial r}+\frac{1}{r^{2}} \frac{\partial^{2} w}{\partial \varphi^{2}}+\frac{\partial^{2} w}{\partial z^{2}}\right]
\end{aligned}
$$

Energy:

$$
u \frac{\partial T}{\partial r}+w \frac{\partial T}{\partial z}=\alpha\left[\frac{\partial^{2} T}{\partial r^{2}}+\frac{1}{r} \frac{\partial T}{\partial r}+\frac{1}{r^{2}} \frac{\partial^{2} T}{\partial \varphi^{2}}+\frac{\partial^{2} T}{\partial z^{2}}\right]
$$

where $p, \rho, v$, and $T$ are the fluid pressure, density, 
kinematic viscosity, and temperature inside the boundary layer and after occurrence of the impingement, respectively. The boundary conditions for the velocity field are:

$$
\begin{aligned}
& u(r, 0)=u(r, 2 \pi), \\
& r=a: \quad w=0, \quad u=-U_{0}(\varphi) .
\end{aligned}
$$

And the two boundary conditions with respect to $\varphi$ are:

$$
\begin{aligned}
& r \rightarrow \infty: \quad w=2 \bar{k} z, \quad u=-\bar{k}\left(r-\frac{a^{2}}{r}\right), \\
& \frac{\partial u(r, 0)}{\partial \varphi}=\frac{\partial u(r, 2 \pi)}{\partial \varphi},
\end{aligned}
$$

in which, Eq. (5) represents no-slip conditions on the cylinder wall and the relations in Eq. (6) show that the viscous flow solution approaches, in a manner analogous to the Hiemenz flow, the potential flow solution as $r \rightarrow \infty$ [12]. This can be confirmed by starting from continuity equation as the following:

$$
-\frac{1}{r} \frac{\partial}{\partial r}(r u) \frac{\partial w}{\partial z}=\text { Constant }=2 \bar{k} z
$$

and integrating it in $r$ and $z$ directions with boundary conditions: $w=0$ when $z=0$; and $u=-U_{0}(\varphi)$ when $r=a$.

For the temperature field, we have:

$$
\begin{array}{ll}
r=a: & T=T_{w}=\text { Constant } \\
r \rightarrow \infty: & T \rightarrow T_{\infty} .
\end{array}
$$

The two boundary conditions with respect to $\varphi$ are:

$$
\begin{aligned}
& T(r, 0)=T(r, 2 \pi), \\
& \frac{\partial T(r, 0)}{\partial \varphi}=\frac{\partial T(r, 2 \pi)}{\partial \varphi},
\end{aligned}
$$

where, $T_{w}$ is the cylinder surface temperature and $T_{\infty}$ is the free-stream temperature.

A reduction in the Navier-Stokes equations is obtained by applying the following similarity transformations:

$$
\begin{aligned}
& u=-\frac{\overline{k a}}{\sqrt{\eta}} f(\eta, \varphi), \quad w=2 \bar{k} f^{\prime}(\eta, \varphi) z, \\
& p=\rho \bar{k}^{2} a^{2} P
\end{aligned}
$$

where $\eta=\left(\frac{r}{a}\right)^{2}$ is dimensionless radial variable. Transformations in Eq. (10) satisfy Eq. (1) automatically and their insertion into Eqs. (2) and (3) yields a coupled system of differential equations in terms of $f(\eta, \varphi)$ and an expression for the pressure:

$$
\begin{aligned}
\eta f^{\prime \prime \prime}+f^{\prime \prime} & +\frac{1}{4 \eta} \frac{\partial^{2} f^{\prime}}{\partial \varphi^{2}}+\operatorname{Re}\left[1+f f^{\prime \prime}-\left(f^{\prime}\right)^{2}\right]=0 \\
P-P_{0}= & -\frac{1}{2}\left(\frac{f^{2}}{\eta}\right)-\left(\frac{f^{\prime}}{\operatorname{Re}}\right) \\
& -\frac{1}{4 \operatorname{Re}} \int_{1}^{\eta} \frac{1}{\eta^{2}} \frac{\partial^{2} f}{\partial \varphi^{2}} d \eta-2\left(\frac{z}{a}\right)^{2}
\end{aligned}
$$

where $\operatorname{Re}=\frac{\bar{k} a^{2}}{2 v}$ is the Reynolds number and prime sign indicates differentiation with respect to $\eta$. From conditions in Eqs. (5)-(7), the boundary conditions for Eqs. (11) and (12) are obtained as follows:

$$
\begin{aligned}
& \eta=1: \quad f^{\prime}(1, \varphi)=0, \quad f(1, \varphi)=S(\varphi), \\
& \eta \rightarrow \infty: \quad f^{\prime}(\infty, \varphi)=1, \\
& f(\eta, 0)=f(\eta, 2 \pi), \quad \frac{\partial f(\eta, 0)}{\partial \varphi}=\frac{\partial f(\eta, 2 \pi)}{\partial \varphi},
\end{aligned}
$$

in which, $S(\varphi)=\frac{U_{0}(\varphi)}{\bar{k} a}$ is the transpiration rate function. Note that Eqs. (11) and (12) are the complete forms of Eqs. (9) and (11) in [15]. These equations are the same if transpiration rate is constant.

For the sake of brevity, only results for the selected values of $S(\varphi)=\cos (\varphi)$ and $S(\varphi)=\cos (2 \varphi)$ are shown in this paper.

To transform the energy equation into a nondimensional form, we introduce:

$$
\theta(\eta, \varphi)=\frac{T(\eta, \varphi)-T_{\infty}}{T_{w}-T_{\infty}} .
$$

Making use of Eqs. (10) and (16) in the energy equation, and with neglecting the small dissipation terms, we have:

$$
\eta \theta^{\prime \prime}+\theta^{\prime}+\frac{1}{4 \eta} \frac{\partial^{2} \theta}{\partial \varphi^{2}}+\operatorname{RePr}\left(f \theta^{\prime}\right)=0
$$

with boundary conditions as:

$$
\begin{aligned}
& \eta=1: \quad \theta(1, \varphi)=1, \\
& \eta \rightarrow \infty: \quad \theta(\infty, \varphi)=0, \\
& \theta(r, 0)=\theta(r, 2 \pi), \quad \frac{\partial \theta(r, 0)}{\partial \varphi}=\frac{\partial \theta(r, 2 \pi)}{\partial \varphi} .
\end{aligned}
$$

Note that Eq. (17) is the complete form of Eq. (14) in [15]. These equations are the same if transpiration rate is constant.

Eqs. (11) and (17), along with boundary conditions (13), (14), (15), (18), and (19), have been 


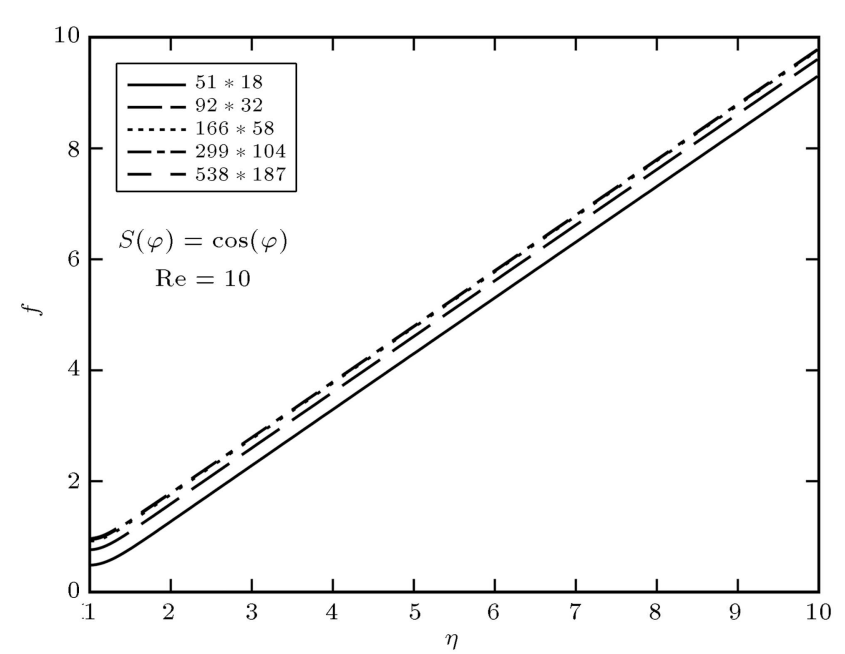

(a)

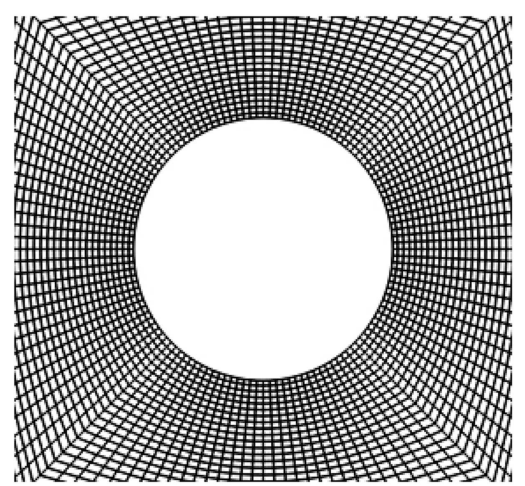

(b)

Figure 2. (a) Sample profiles of $f(\eta, \varphi)$ distributions on the cylinder for various meshsizes at $\operatorname{Re}=10$ and $S(\varphi)=\cos (\varphi)$. (b) Sample of grid system.

solved numerically by an implicit, iterative tri-diagonal finite difference method similar to that discussed by Blottner [33]. To assess the grid independence of the numerical scheme, the distributions of the function $f(\eta, \varphi)$ against $\eta$ on the cylinder were initially tested with different $(\eta, \varphi)$ mesh sizes of $51 * 18,92 * 32$, $166 * 58,299 * 104$, and $538 * 187$ in Figure $2(\mathrm{a})$. In this set of mesh sizes, as can be seen, the coefficient of 1.8 was used in each test to increase the number of mesh grids in both directions of $\eta$ and $\varphi$. It was found that the variations of the function distributions, $f(\eta, \varphi)$, on the cylinder were not significant between $(\eta, \varphi)$ and mesh sizes of $(166 * 58),(299 * 104)$, and $(538 * 187)$. Hence, a grid of $(299 * 104)$ in $\eta-\varphi$ directions was applied for the computational domain in the cylinder. Fine, non-uniform grid spacing was used in the $\eta$-direction to capture the rapid changes, such as grid lines, being closer packed near the walls. On the other hand, a uniform mesh was implemented in the $\varphi$-direction. Figure 2(b) illustrates a sample of computational meshes used in this investigation [34].

\section{Shear-stress}

The shear-stress at the cylinder surface is calculated from [12]:

$$
\sigma=\mu\left[\frac{\partial w}{\partial r}\right]_{r=a}
$$

where $\mu$ is the fluid viscosity. Using Eq. (10), the shear stress at the cylinder surface for semi-similar solutions becomes:

$$
\sigma=\mu \frac{2}{a}\left[2 \bar{k} z f^{\prime \prime}(1, \varphi)\right] \Rightarrow \frac{\sigma a}{4 \mu \bar{k} z}=f^{\prime \prime}(1, \varphi) .
$$

Results for $\sigma a / 4 \mu \bar{k} z$ against $\varphi$ for different values of Reynolds number and transpiration rate functions will be presented later.

\section{Heat transfer coefficient}

The local heat transfer coefficient and rate of heat transfer for the defined wall temperature case are given by:

$$
h=\frac{q_{w}}{T_{w}-T_{\infty}}=\frac{-k\left(\frac{\partial T}{\partial r}\right)_{r=a}}{T_{w}-T_{\infty}}=-\frac{2 k}{a} \frac{\partial \theta(1, \varphi)}{\partial \eta},
$$

where:

$$
q_{w}=-\frac{2 k}{a} \frac{\partial \theta(1, \varphi)}{\partial \eta}\left(T_{w}-T_{\infty}\right)
$$

and in terms of Nusselt number:

$$
\mathrm{Nu}=\frac{h a}{2 k}=-\theta^{\prime}(1, \varphi)
$$

\section{Presentation of results}

In this section, solution of semi-similar Eqs. (11) and (17) along with surface shear stresses for prescribed values of surface temperature, with the selected values of Reynolds and Prandtl numbers and transpiration rate function, are presented. For the sake of brevity, only results for selected values of $S(\varphi)=\cos (\varphi)$ and $S(\varphi)=\cos (2 \varphi)$ are shown in this paper:

a) $\boldsymbol{S}(\varphi)=\cos (\varphi)$ : Effects of transpiration rate function on function $f(\eta, \varphi)$ against $\varphi$ for $\operatorname{Pr}=0.7$ and selected value of Reynolds number, $\operatorname{Re}=1.0$, are shown in Figure 3. In this figure, negative $S(\varphi)$ is blowing rate and positive $S(\varphi)$ is the suction rate. It is evident from this figure that as transpiration rate function increases, the $f$ function increases and if $S(\varphi)$ decreases, the $f$ function decreases. It is interesting to note that as $S(\varphi)$ increases, the depth of diffusion of the fluid velocity field in radial direction increases. Sample profiles of 


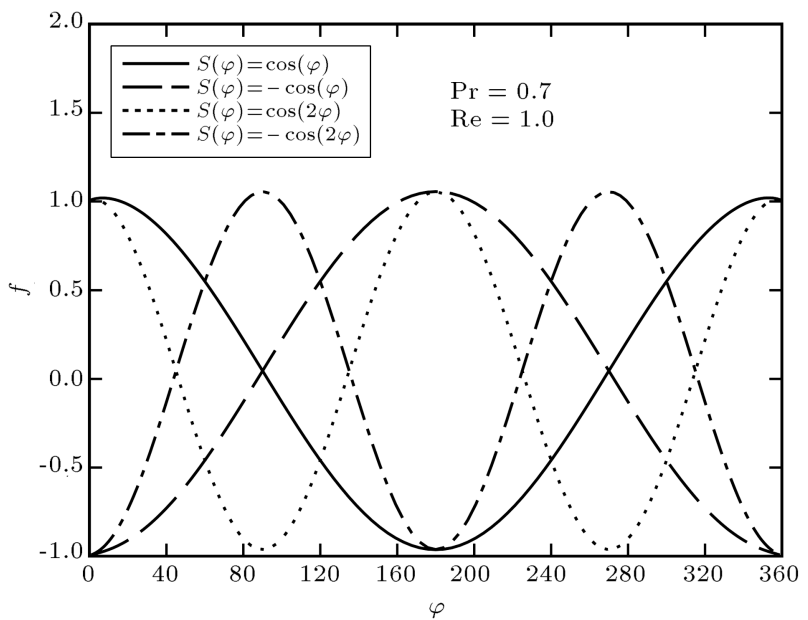

Figure 3. Variations of $f(\eta, \varphi)$ in terms of $\eta$ and $\varphi$ at $\operatorname{Pr}=0.7$ and $\operatorname{Re}=1.0$, for different values of transpiration rate function.

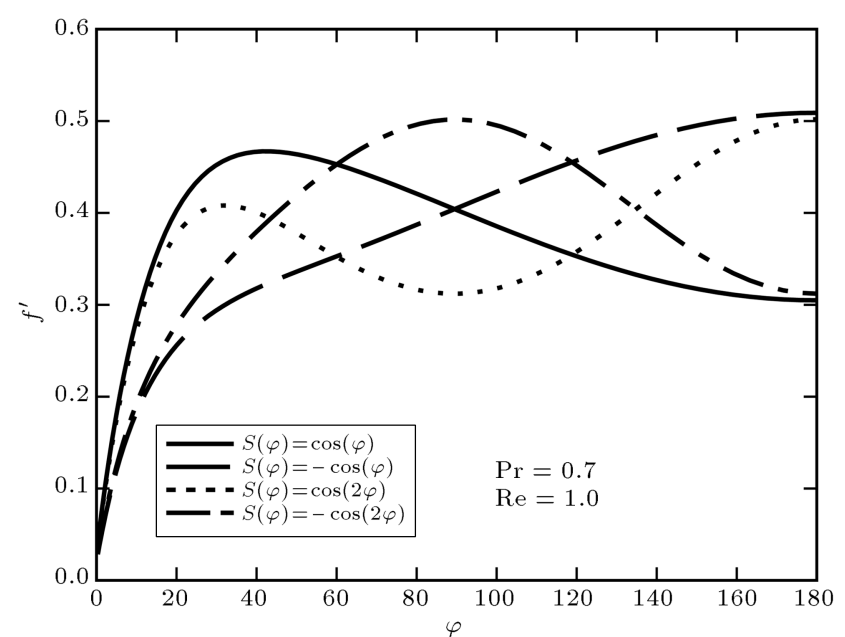

Figure 4. Variations of $f^{\prime}(\eta, \varphi)$ in terms of $\eta$ and $\varphi$ at $\operatorname{Pr}=0.7$ and $\operatorname{Re}=1.0$, for different values of transpiration rate function.

the $f^{\prime}(\eta, \varphi)$ function in terms of $\varphi$ are depicted in Figure 4 for selected values of Reynolds number and transpiration rate function. From this figures, the initial slope of the $f^{\prime}$ function increases with increasing Reynolds number and transpiration rate function. In fact, the increase in transpiration rate function and Reynolds number decreases the thickness of the boundary layer. Effect of variation of Prandtl number and Reynolds number on $\theta(\eta, \varphi)$ function in terms of $\varphi$ for the case of constant surface temperature and $S(\varphi)=\cos (\varphi)$ is shown in Figures 5 and 6 . As Prandtl number and Reynolds number increase, the depth of diffusion of the thermal boundary layer decreases and therefore, the heat-transfer coefficient increases. Sample profiles of the $\theta(\eta, \varphi)$ function in terms of $\varphi$ for $\operatorname{Re}=1.0$ and $\operatorname{Pr}=0.7$ are presented in Figure 7 for selected values of transpiration rate function. It is seen that

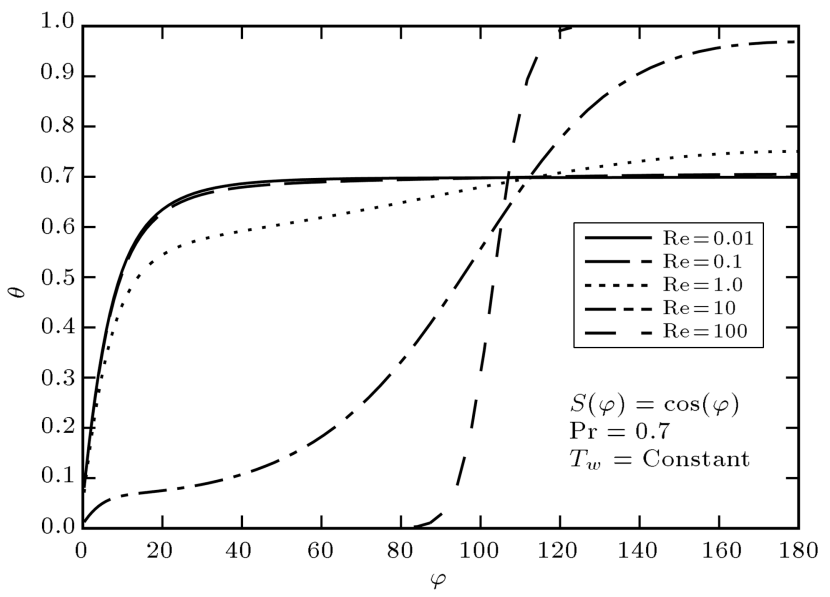

Figure 5. Variations of $\theta(\eta, \varphi)$ in terms of $\eta$ and $\varphi$ at $\operatorname{Pr}=0.7$ and $S(\varphi)=\cos (\varphi)$, for different values of Reynolds number.

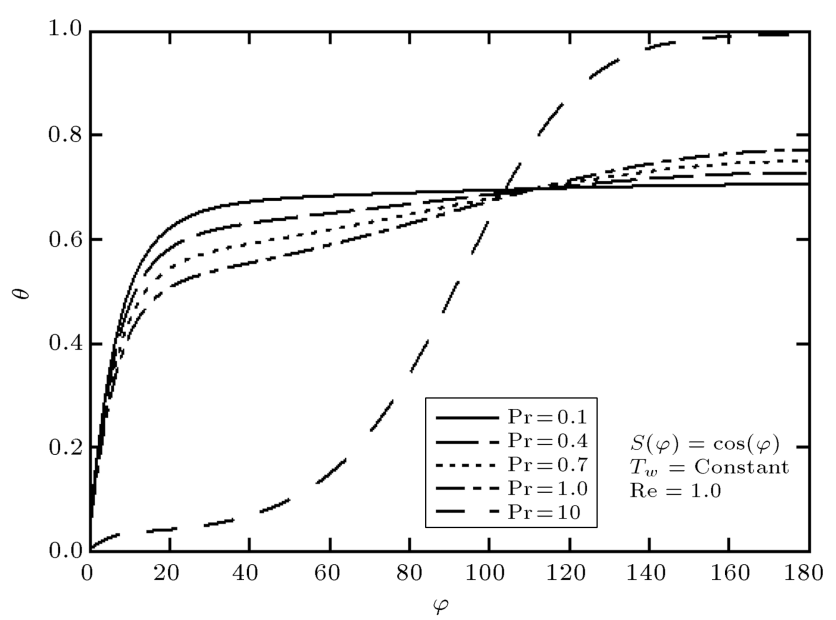

Figure 6. Variations of $\theta(\eta, \varphi)$ in terms of $\eta$ and $\varphi$ at $\operatorname{Re}=1.0$ and $S(\varphi)=\cos (\varphi)$, for different values of Prandtl number.

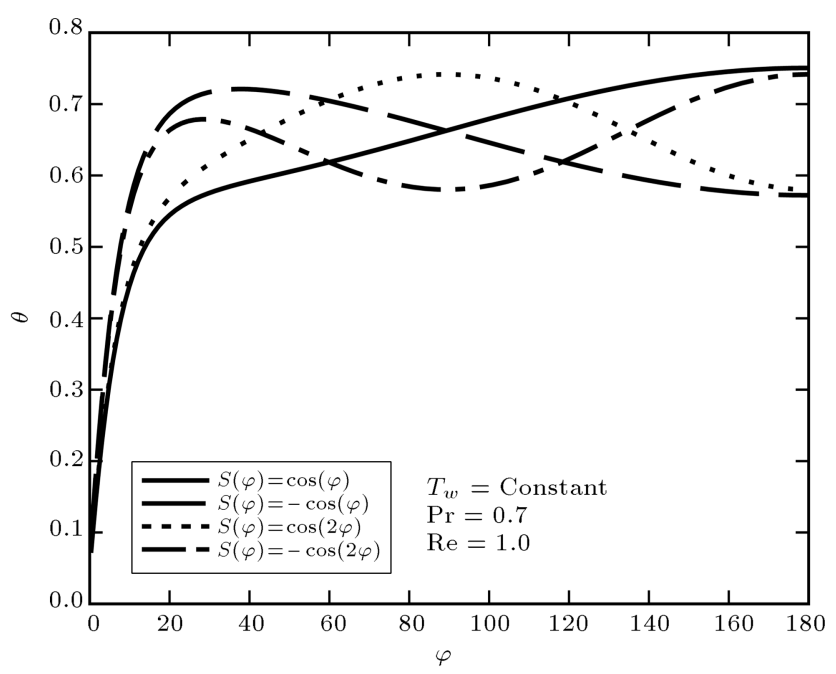

Figure 7. Variations of $\theta(\eta, \varphi)$ in terms of $\eta$, and $\varphi$ at $\operatorname{Pr}=0.7$ and $\operatorname{Re}=1.0$, for different values of transpiration rate function. 
as the rate of transpiration rate function increases, the depth of diffusion of the temperature field decreases and thus, the heat transfer coefficient increases. Sample profiles of surface shear stress against $\varphi$ for $\operatorname{Re}=1.0, \operatorname{Pr}=0.7$, and $S(\varphi)=$ $\cos (\varphi)$ are shown in Figure 8 for selected values of Reynolds number and transpiration rate function. The increase in transpiration rate function and Reynolds Number increases the wall shear stress in $\varphi$ direction and on the other hand causes the value of fluid velocity in this direction to approach its value in inviscid flow, rapidly. In fact, the increase in transpiration rate function and Reynolds number decreases the thickness of the boundary layer. Sample profiles of the Nusselt number (local heat transfer coefficient) for $\operatorname{Re}=1.0, \operatorname{Pr}=0.7$, and $S(\varphi)=\cos (\varphi)$ are shown in Figure 9 for selected

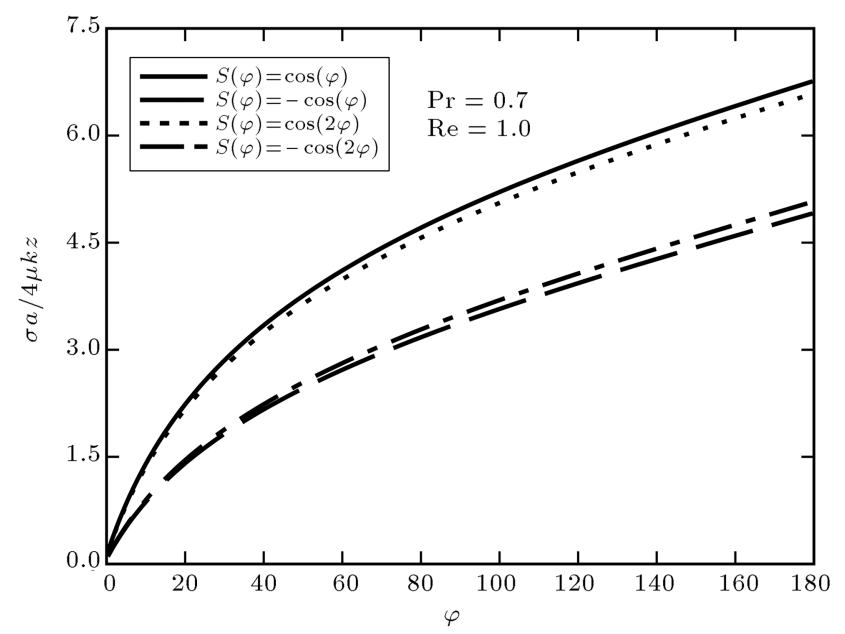

Figure 8. Variations of shear stress in terms of $\varphi$ for different values of Reynolds number, and different values of transpiration rate function.

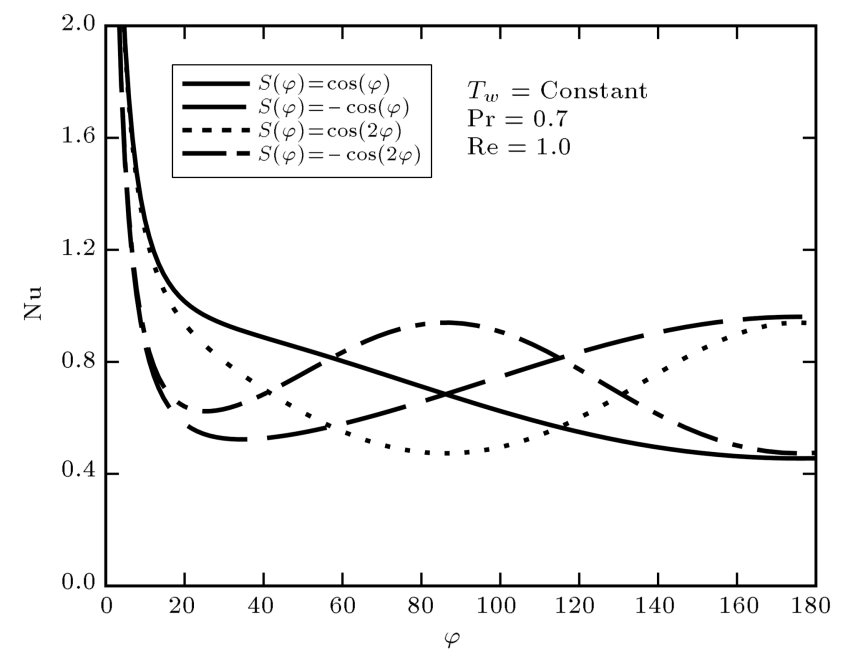

Figure 9. Variations of Nusselt number in terms of $\varphi$ for different values of Prandtl number, and different values of transpiration rate function. values of Prandtl number and transpiration rate function. Nusselt number increases as Prandtl number and transpiration rate function increase.

b) $\boldsymbol{S}(\varphi)=\cos (2 \varphi)$ : Sample profiles of surface shear stress against $\varphi$ for $\operatorname{Pr}=0.7$ and $S(\varphi)=\cos (2 \varphi)$ (drawn in Figure 10) are shown in Figure 11 for selected values of Reynolds number. The increase in Reynolds number increases the wall shear stress in $\varphi$ direction and on the other hand, causes the value of fluid velocity in this direction to approach its value in inviscid flow, rapidly. In fact, the increase in Reynolds number decreases the thickness of the boundary layer. Sample profiles of the Nusselt number (local heat transfer coefficient) for $\operatorname{Re}=1.0$ and $S(\varphi)=\cos (2 \varphi)$ are shown in Figure 12 for selected values of Prandtl number. Nusselt number increases as Prandtl number increases. To assist the fluid flow behavior, streamlines have been plotted in Figure 13 for

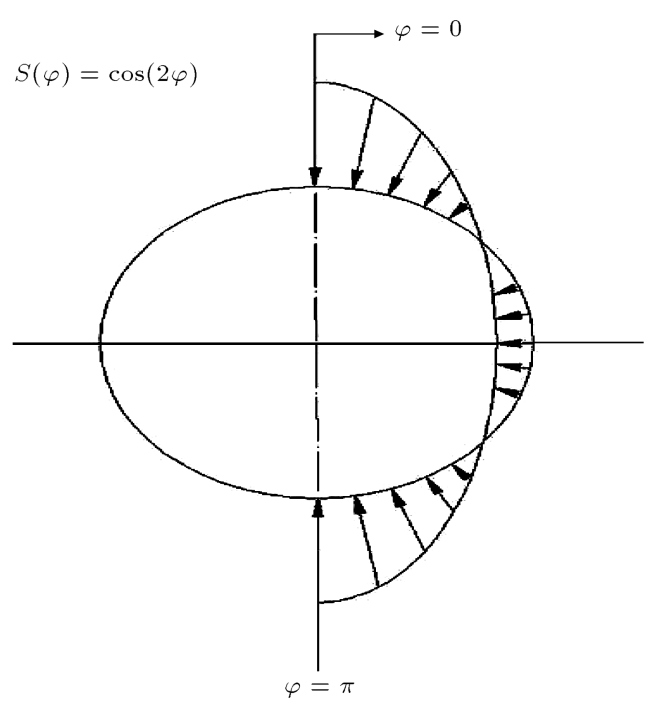

Figure 10. Schematic diagram of the transpiration function, $S(\varphi)=\cos (2 \varphi)$.

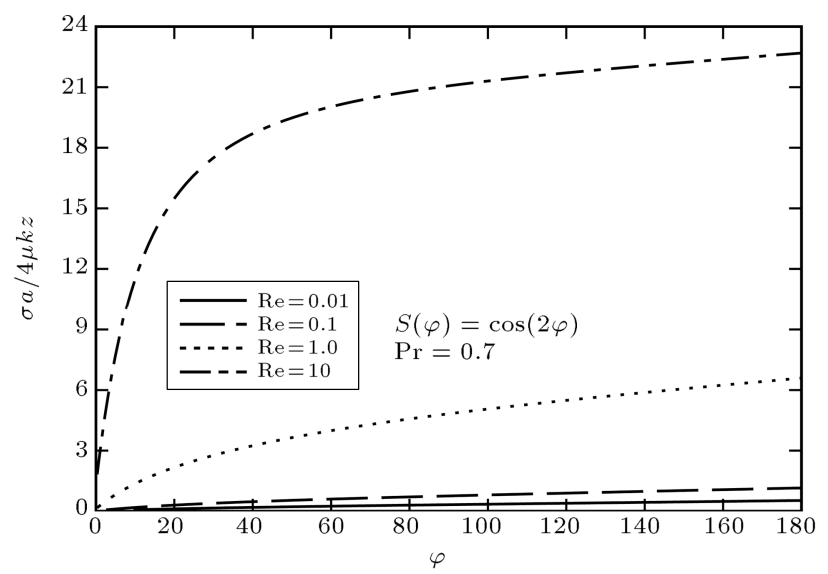

Figure 11. Variations of shear stress in terms of $\varphi$ for different values of Reynolds number. 


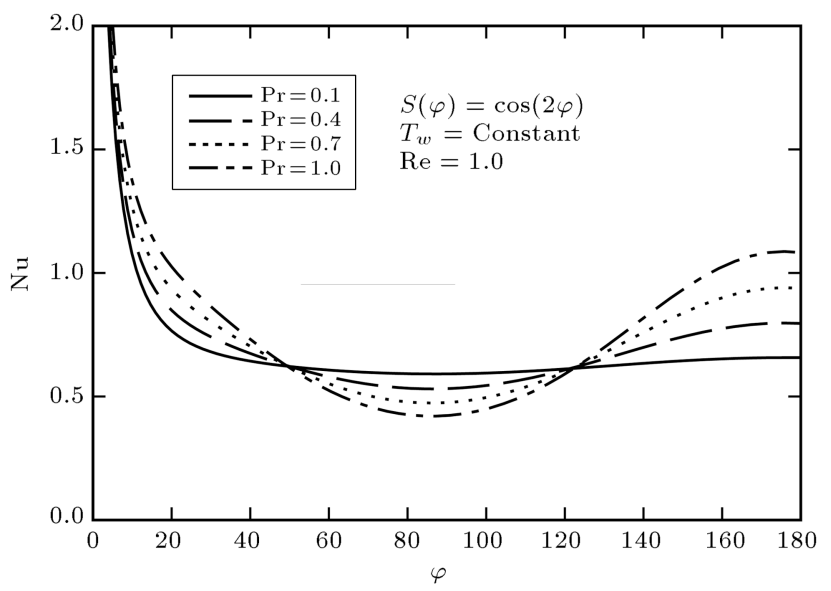

Figure 12. Variations of shear stress in terms of $\varphi$ for different values of transpiration rate function.

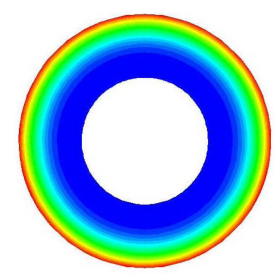

(a)

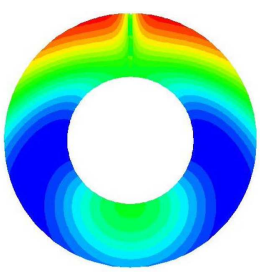

(b)

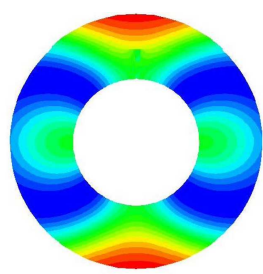

(c)
Figure 13. Variations of streamlines for different values of transpiration rate function at $\operatorname{Re}=10:($ a) $S(\varphi)=0$; (b) $S(\varphi)=\cos (\varphi)$; and (c) $S(\varphi)=\cos (2 \varphi)$.

different values of transpiration rate function at $S(\varphi)=0, S(\varphi)=\cos (\varphi), S(\varphi)=\cos (2 \varphi)$, and $\operatorname{Re}=10$.

\section{Conclusions}

The steady-state viscous flow and heat transfer in the vicinity of a non-axisymmetric stagnation point of an infinite stationary cylinder with non-uniform normal transpiration $U_{0}(\varphi)$ and constant wall temperature have been investigated. Numerical solutions of the Navier-Stokes equations and energy equation have been derived in this problem. A reduction in these equations has been obtained by use of appropriate similarity transformations. The semi-similar solution of the Navier-Stokes equations and energy equation has been obtained numerically using an implicit finitedifference scheme. All the solutions above have been presented for Reynolds number ranging from 0.01 to 100 for different values of Prandtl number and for selected values of transpiration rate function. For all transpiration rate functions, with increase in Reynolds number, both components of the velocity field increase and for all transpiration rate functions, with increase in Prandtl number, the temperature field decreases. Dimensionless shear stresses corresponding to all the cases increase with increase in Reynolds number and transpiration rate function. The local coefficient of heat transfer (Nusselt number) increases with the increasing transpiration rate function and Prandtl number. For the case of axisymmetric stagnation-point flow, $f=f(\eta), f^{\prime}=f^{\prime}(\eta)$, and $S(\varphi)=0$ or $\frac{\partial^{2} f}{\partial \varphi^{2}}=0$, $\frac{\partial^{2} f^{\prime}}{\partial \varphi^{2}}=0$, and $\frac{\partial^{2} \theta}{\partial \varphi^{2}}=0$, and similarity variables and components of velocity by Wang [5], as well as energy equation by Gorla [7] and Saleh and Rahimi [15], are reached.

\section{References}

1. Hiemenz, K. "Die Grenzschicht an einem in den gleichförmingen Flüssigkeitsstrom eingetauchten geraden Kreiszylinder", Dinglers Polytechnisches Journal, 326, pp. 321-410 (1911).

2. Homann, F.Z. "Der Einfluss Grosser Zahighkeit bei der Strmung um den Zylinder und um die Kugel", Zeitschrift für Angewandte Mathematik und Mechanik, 16, pp. 153-164 (1936). DOI:10.1002/zamm.19360160304

3. Howarth, L. "The boundary layer in three-dimensional flow. Part 2. The flow near stagnation point", Philosophical Magazine, 42(7), pp. 1433-1440 (1951).

4. Davey, A. "Boundary-layer flow at a saddle point of attachment", Journal of Fluid Mechanics, 10(4), pp. 593-610 (1961). DOI: 10.1017/S0022112061000391

5. Wang, C.Y. "Axisymmetric stagnation flow on a cylinder", Quarterly of Applied Mathematics, 32, pp. 207213 (1974).

6. Wang, C.Y. "Axisymmetric stagnation flow towards a moving plate", American Institute of Chemical Engineers Journal, 19(5), pp. 1080-1082 (1973). DOI: 10.1002 /aic. 690190540

7. Gorla, R.S.R. "Heat transfer in an axisymmetric stagnation flow on a cylinder", Applied Scientific Research, 32(5), pp. $541-553$ (1976). DOI: 10.1007/BF00385923

8. Gorla, R.S.R. "Unsteady laminar axisymmetric stagnation flow over a circular cylinder", Developments in Mechanics, 9, pp. 286-288 (1977).

9. Gorla, R.S.R. "Non-similar axisymmetric stagnation flow on a moving cylinder", International Journal of Engineering Science, 16(6), pp. 392-400 (1978).

10. Gorla, R.S.R. "Transient response behavior of an axisymmetric stagnation flow on a circular cylinder due to time-dependent free stream velocity", Letters in Applied and Engineering Sciences; An International Journal, 16(7), pp. 493-502 (1978). Doi:10.1016/0020$7225(78) 90082-4$

11. Gorla, R.S.R. "Unsteady viscous flow in the vicinity of an axisymmetric stagnation-point on a cylinder", International Journal of Engineering Science, 17(1), pp. 87-93 (1979). DOI:10.1016/0020-7225(79)90009-0

12. Cunning, G.M., Davis, A.M.J. and Weidman, P.D. "Radial stagnation flow on a rotating cylinder 
with uniform transpiration", Journal of Engineering Mathematics, 33(2), pp. 113-128 (1998). DOI: 10.1023/A:1004243728777

13. Grosch, C.E. and Salwen, H. "Oscillating stagnation point flow", Proceedings of Royal Society of London, Series A: Mathematical and Physical Sciences, 384(1786), pp. 175-190 (1982).

14. Takhar, H.S., Chamkha, A.J. and Nath, J. "Unsteady axisymmetric stagnation-point flow of a viscous fluid on a cylinder", International Journal of Engineering Science, 37(15), pp. 1943-1957 (1999). DOI: 10.1016/S0020-7225(99)00009-9

15. Saleh, R. and Rahimi, A.B. "Axisymmetric stagnationpoint flow and heat transfer of a viscous fluid on a moving cylinder with time-dependent axial velocity and uniform transpiration", Journal of Fluids Engineering, 126(6), pp. 997-1005 (2004). DOI: 10.1115/1.1845556

16. Rahimi, A.B. and Saleh, R. "Axisymmetric stagnationpoint flow and heat transfer of a viscous fluid on a rotating cylinder with time-dependent angular velocity and uniform transpiration", Journal of Fluids Engineering, 129(1), pp. 107-115 (2007).

17. Rahimi, A.B. and Saleh, R. "Similarity solution of unaxisymmetric heat transfer in stagnation-point flow on a cylinder with simultaneous axial and rotational movements", Journal of Heat Transfer, 130(5), pp. 054502-1-054502-5 (2008).

18. Abbasi, A.S. and Rahimi, A.B. "Non-axisymmetric three- dimensional stagnation-point flow and heat transfer on a flat plate", Journal of Fluids Engineering, 131(7), pp. 074501.1-074501.5 (2009).

19. Abbasi, A.S. and Rahimi, A.B. "Three-dimensional stagnation-point flow and heat transfer on a flat plate with transpiration", Journal of Thermophysics and Heat Transfer, 23(3), pp. 513-521 (2009). DOI: 10.2514/1.41529

20. Abbasi, A.S., Rahimi, A.B. and Niazman, H. "Exact solution of three-dimensional unsteady stagnation flow on a heated plate", Journal of Thermophysics and Heat Transfer, 25(1), pp. 55-58 (2011). DOI: 10.2514/1.48702

21. Abbasi, A.S. and Rahimi, A.B. "Investigation of twodimensional stagnation-point flow and heat transfer impinging on a flat plate", Journal of Heat Transfer, accepted as Technical Brief, 134, pp. 1-5 (June 2012).

22. Subhashini, S.V. and Nath, G. "Unsteady compressible flow in the stagnation region of two-dimensional and axisymmetric bodies", Acta Mechanica, 134(3-4), pp. 135-145 (1999). DOI: 10.1007/BF01312652

23. Kumari, M. and Nath, G. "Unsteady compressible 3dimensional boundary layer flow near an axisymmetric stagnation point with mass transfer", International Journal of Engineering Science, 18(12), pp. 1285-1300, (1980). DOI: 10.1016/0020-7225(80)90120-2

24. Kumari, M. and Nath, G. "Self-similar solution of unsteady compressible three-dimensional stagnationpoint boundary layers", Journal of Applied Mathematics and Physics, 32(3) (1981).
25. Katz, A. "Transformations of the compressible boundary layer equations", SIAM Journal on Applied Mathematics, 22(4) (1972).

26. Afzal, N. and Ahmad, S. "Effect of transpiration and injection on self- similar solutions of second-order boundary layer equations", International Journal of Heat and Mass Transfer, 18(5), pp. 607-614 (1975). DOI: $10.1016 / 0017-9310(75) 90272-0$

27. Libby, P.A. "Heat and mass transfer at a general threedimensional stagnation point", AIAA Journal, 5(3), pp. 507-517 (1967). DOI: $10.2514 / 3.4008$

28. Gersten, K., Papenfuss, H.D. and Gross, J.F. "Influence of the Prandtl number on second-order heat transfer due to surface curvature at a three-dimensional stagnation point", International Journal of Heat and Mass Transfer, 21(3), pp. 275-284 (1978). DOI: 10.1016/0017-9310(78)90120-5

29. Mohammadiun, H. and Rahimi, A.B. "Stagnationpoint flow and heat transfer of a viscous, compressible fluid on a cylinder", Journal of Thermophysics and Heat Transfer, 26(3), pp. 494-502 (2012).

30. Mohammadiun, H., Rahimi, A.B. and Kianifar, A. "Axisymmetric stagnation-point flow and heat transfer of a viscous, compressible fluid on a cylinder with constant heat flux", Scientia Iranica, 20(1), pp. 185194 (2013).

31. Alizadeh, R., Rahimi, A.B. and Najafi, M. "Unaxisymmetric stagnation-point flow and heat transfer of a viscous fluid on a moving cylinder with time-dependent axial velocity", Journal of the Brazilian Society of Mechanical Sciences and Engineering, 38(85), p. 98 (2016). DOI: $10.1007 / \mathrm{s} 40430-015-0389-2$

32. Schlichting, H., Boundary Layer Theory, McGraw Hill Book Company Inc., New York (1968).

33. Blottner, F.G. "Finite-difference methods of solution of the boundary layer equations", AIAA. J., 8, pp. 193-205 (1970).

34. Mozayyeni, H.R. and Rahimi, A.B. "Mixed convection in cylindrical annulus with rotating outer cylinder and constant magnetic field with an effect in the radial direction", Scientia Iranica, 19(1), pp. 91-105 (2012).

\section{Biographies}

Rasool Alizadeh was born in Quchan, Iran, in 1982. He received his BS degree in Mechanical Engineering from Azad University of Mashhad, Mashhad, Iran, in 2006 and his MS degree in Mechanical Engineering from Guilan University, Rasht, Iran, in 2009. He is currently a PhD Student in Mechanical Engineering, Science and Research Branch of Islamic Azad University, Tehran, Iran.

Asghar B. Rahimi was born in Mashhad, Iran, in 1951. He received his BS degree in Mechanical Engineering from Tehran Polytechnic, Iran, in 1974, 
and $\mathrm{PhD}$ degree in Mechanical Engineering from the University of Akron, Ohio, USA, in 1986. He has been Professor in the Department of Mechanical Engineering at Ferdowsi University of Mashhad since 2001. His research and teaching interests include heat transfer and fluid dynamics, gas dynamics, continuum mechanics, applied mathematics, and singular perturbation.

Mohammad Najafi was born in 1957 in Tehran, Iran.
He received his $\mathrm{PhD}$ degree in Mechanical Engineering, Thermal Fluids, in 1989 from the University of Alabama, USA. Since then, he was a full-time faculty member in Mechanical Engineering Department at University of Louisiana, USA, until 1996, when he was hired at Tehran Science and Research Branch of Islamic Azad University in Iran. He has been a full-time Thermal Fluids Faculty member in the Mechanical Engineering Department since then. 